\title{
Pathological Investigations into the Tooth Germs and Related Tissues in a Thalidomide-Baby
}

\author{
by \\ Yozo KOBAYAKAWA* and Youch KURIHARA**
}

Incidence of the so-called thalidomide baby has created a gravest social problem and it is typical of the collateral effect to be derived from various new drugs which have been more or less fashionable in recent years. We were fortunate enough to obtain for our investigatory purposes the jaws from a thalidomide-baby (Contergan baby, to use the term coined by LENZ[1]) which had been born of a woman who had taken a large dosage of thalidomide preparations in the course of her pregnancy. The present paper is concerned with some of its interesting findings pathologically examined.

\section{A. Subject :}

The subject was a malformed baby born of a 21-year-old mother (her last menstruation was on the 25th January, 1962) as her first child. The mother began to take thalidomide preparation (Pro-Ban M) to ease her hyperemesis gravidarum in the amount of 3 to 4 tablets a day for the period of 75 days, the total consumption amounting to $1,392 \mathrm{mg}$. On 10th October of the same year, i. e., after 41 weeks and 1 day of

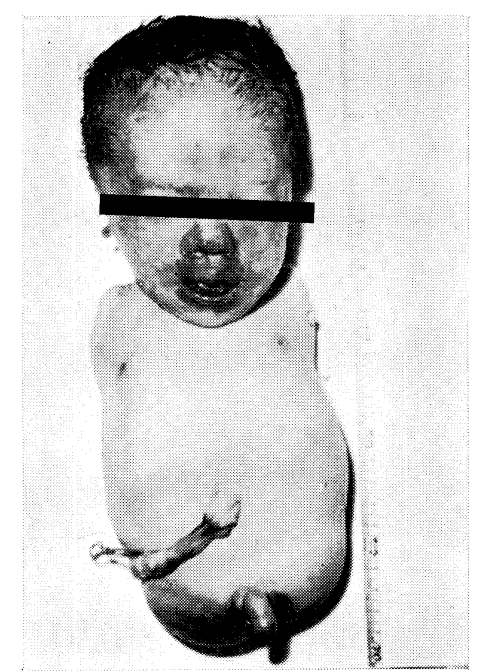

Fig. 1

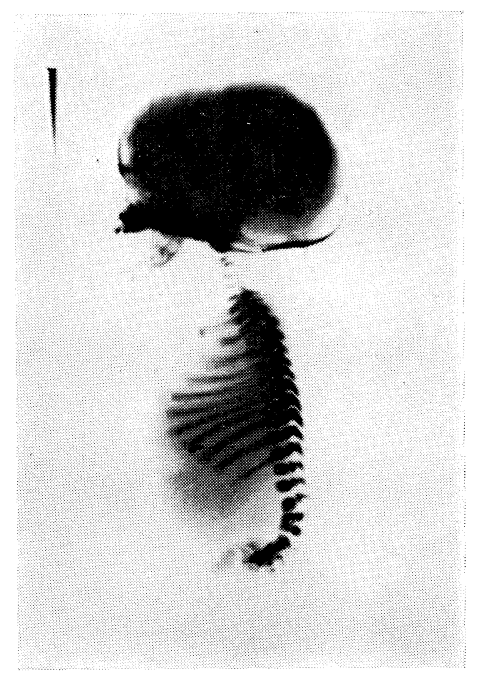

Fig. 2

\footnotetext{
* 小早川 庸造：Dept. of Pathology, Nihon Univ. School of Dentistry.

** 栗原 洋一: Dept. of Pedodontics, Nihon Univ. School of Dentistry.

Substance of the present paper was presented before the 15th Nihon University Dental Meeting held on May 25th, 1963.
} 


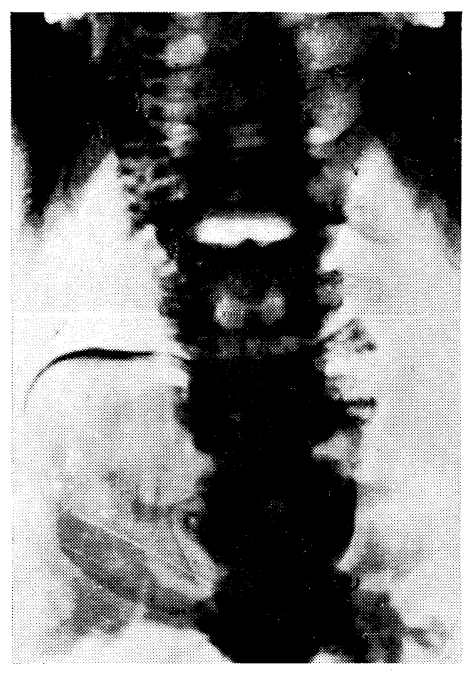

Fig. 3

pregnancy, she was delivered of a boy. The boy was $1,852 \mathrm{~g}$ and measured $26,5 \mathrm{~cm}$ in stature at the time of birth and he died soon after birth. The familial record did not register the fact of a malformed baby neither on the paternal nor the maternal side. As is shown by Fig. 1, the baby lacked the extremities and ears and there was the retentio testis in an abdominal cavity. In addition to these, a large area of hemangioma on the nose-tip, nosewing and around the mouth. The roentgenographic examination revealed complete amelia and the missing of a left ilium (Figs. 2, 3).

\section{B. Macroscopic Findings of the Jaws :}

Except for a circumscribed corrugated swelling of the palatine membrane in the median region of the upper jaw, there were no pathological changes worthy of note (Fig. 4).

\section{Roentgenographic Findings of the Jaws.}

No changes were detected in the number of tooth germs and their shapes in the upper jaw, while in the lower jaw some morphological changes were apparent as regards both lateral incisors. That is, a wedge-like interstice developed in the center of the cutting edge of the left incisor giving an appearance of a confused tooth and the right one was in the shape of a conic (Figs. 5, 6).

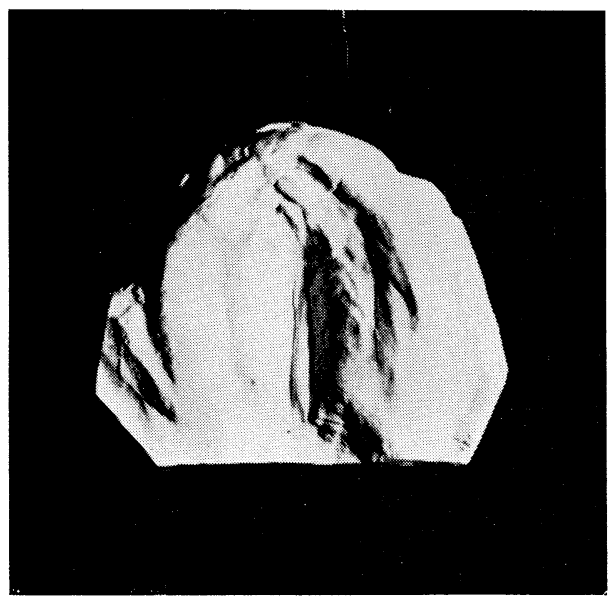

Fig. 4

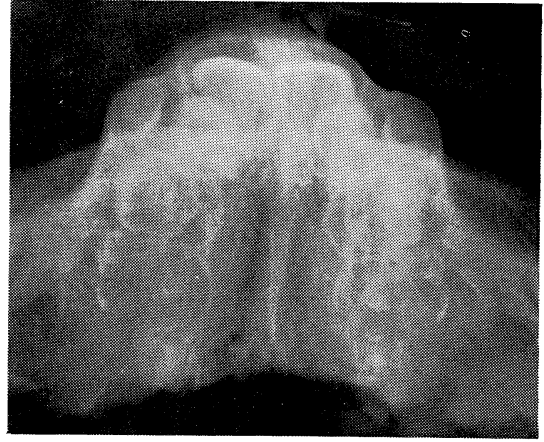

Fig. 5

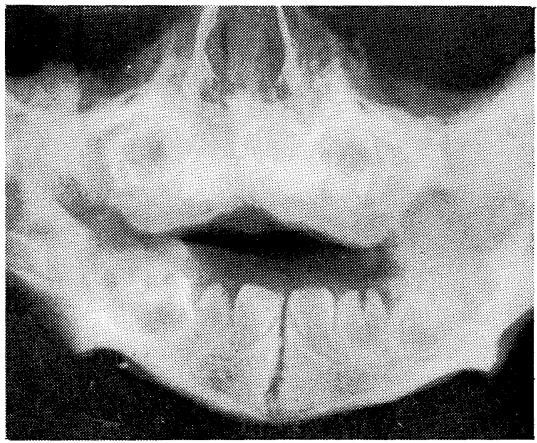

Fig. 6 


\section{Histological Findings of the Jaws.}

After the macroscopic and roentgenographic examinations of the subject had been performed in the above manner, the jaws were made decalcified and prepared into celloidin imbedded specimens. After they were stained with hematoxylineosin, the specimens were subjected to microscopic examination. The following points received attention in microscopy.

\section{Oral mucosa.}

No changes of particular importance were seen.

\section{Enamel.}

Although the general state of calcification did not differ from the normal condition to any appreciable extent, changes were observable in spots where the surface was roughened like waves. In these spots enough contrast was developed in the shade of stain, testifying to an uneven state of calcification. Changes were also observed in the enamel epithelial cells bordering these spots. This will be taken up more in detail in the ensuing lines (Figs. 7, 8, 9).

\section{Enamel epithelial cells.}

In that part of the enamel epithelium where enamel organ involving enamel pulp was preserved there was detected morphological change. On the other hand, however, desquamation from the enamel surface was observed in the united enamel epithelium in the cutting edge or cusp region, in which some liquid lent itself to eosin-staining readily. One observed a hyaline droplet-like matter and minor cells in this liquid. Ameloblasts in this area were remarkably enlarged and a kind of

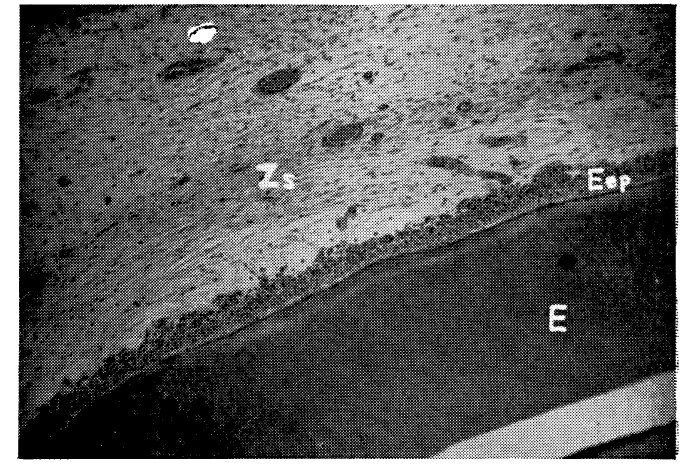

Fig. 7

$\mathrm{E}$ enamel

Eep enamel epithelium

$\mathrm{Zs}$ tooth sac

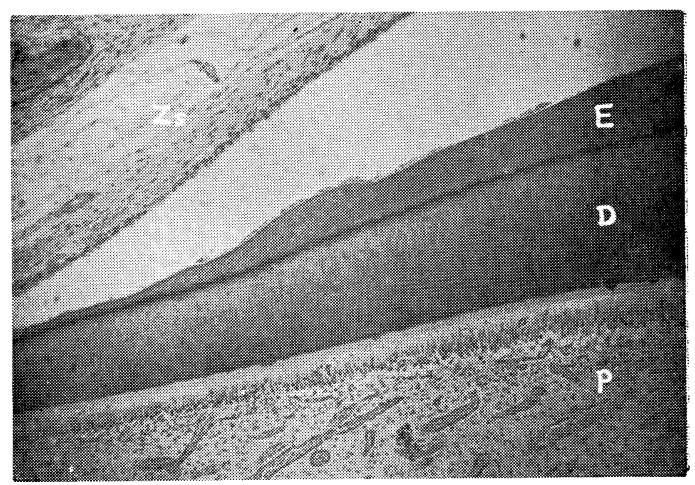

Fig. 8

P dental pulp

D dentin

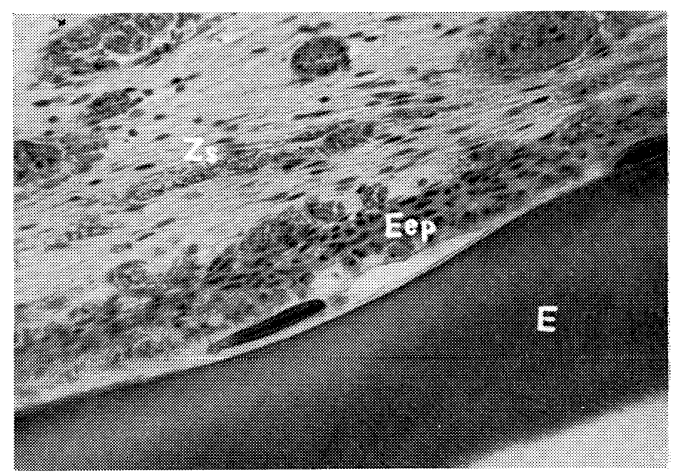

Fig. 9

E enamel

Eep enamel epithelium

$\mathrm{Zs}$ tooth sac 


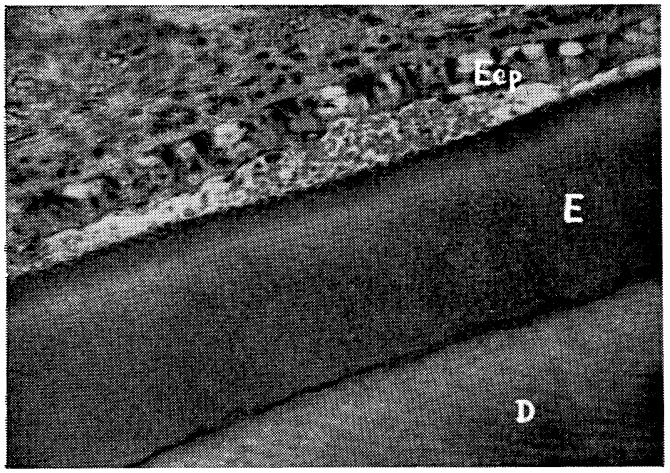

Fig. 10. Desquamation of enamel epithelium Eep enamel epithelium $\mathrm{E}$ enamel D dentin

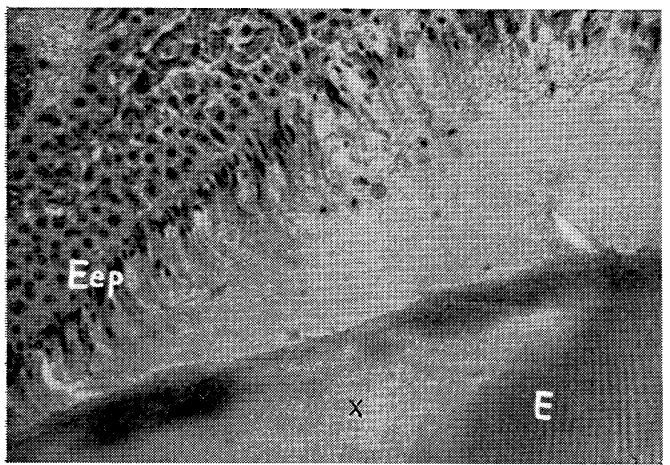

Fig. 11. Desquamation of enamel epithelium

Eep enamel epithelium

$\mathrm{E}$ enamel

D dentin

$\times$ marks hypomineralization

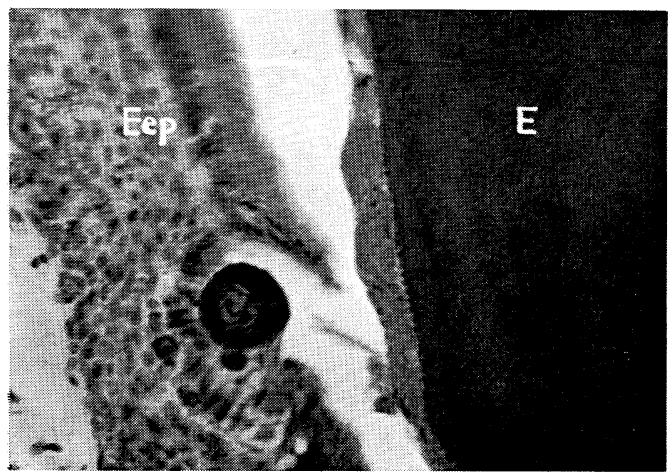

Fig. 12. Formation of enamel nodule clear in appearance. The cells adjacent to this area were oppressed into stick-like forms and their nuclei pyknotic. The formation of enamel nodule was partially noted (Figs. 10, 11, 12).

\section{Dentin.}

No changes were observed in dentin altogether.

\section{Dental pulp.}

Hyperemia of a high degree had taken place in the tissues next to the dental pulp, and here and there one noted bleeding and edemas. In the odontoblastic layer there was seen a vascular degeneration (Figs. 13, 14, 15).

6. Tooth sac.

Hyperemia, bleeding and edemic changes were extensively observed all over the tooth sacs (Figs. $16,17)$.

7. Jaw bones and bone-marrow.

Although no change was apparent in the bone structure itself,

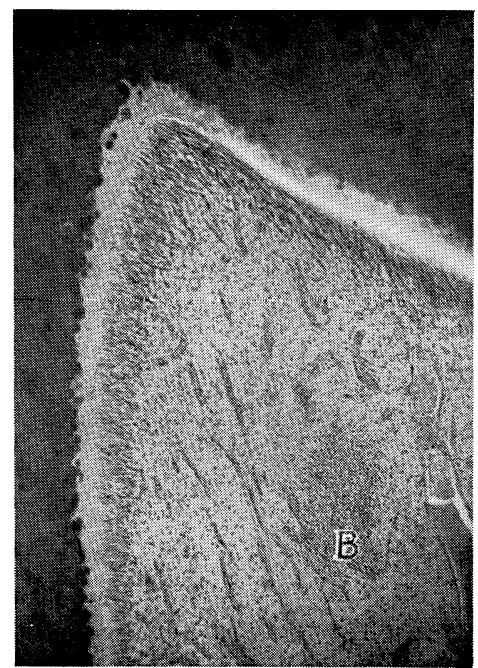

Fig. 13

B bleeding spot 


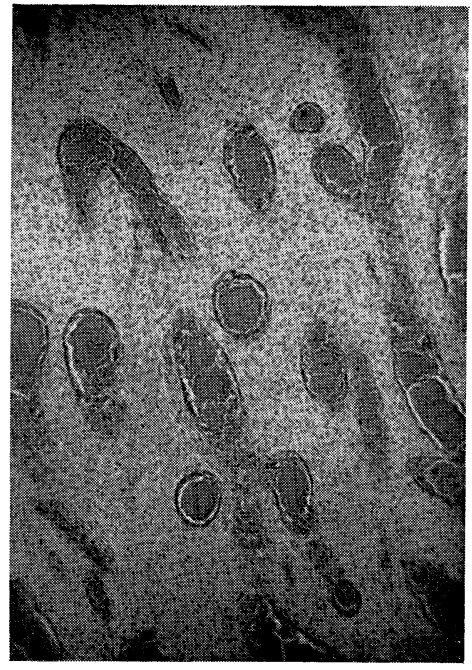

Fig. 14. Congestion of the dental pulp

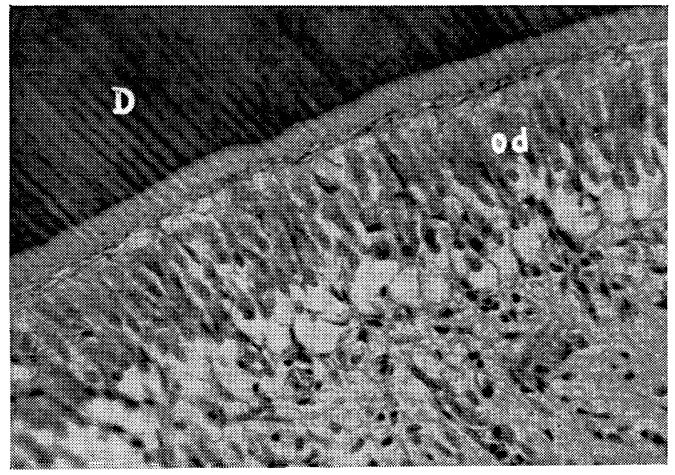

Fig. 15

Od vascular degeneration in the odontoblastic layer

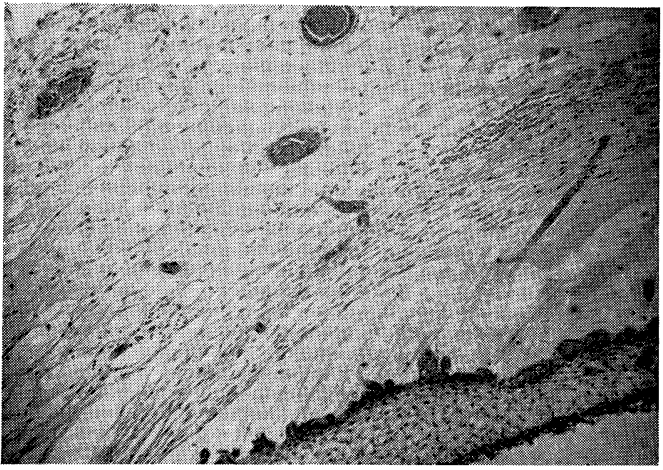

Fig. 16. A severe edemic change in the tooth sac region

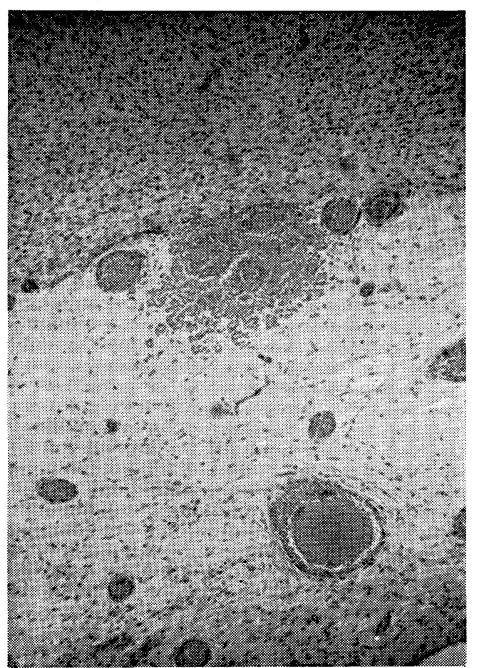

Fig. 17. Blood congestion and edemic change in the tooth sac region

hyperemia of a severe nature was observed in the bone-marrow with the blood vessels being enlarged and full. The bone-marrow cavity was filled with this kind of blood vessels, giving an appearance of angioma. Bleeding and edemas were observed here and there (Fig. 18).

\section{Membranes of accessory nasal} cavity.

In this part of nasal membranes there were noted an intense bleeding and hyperemia in the appearance

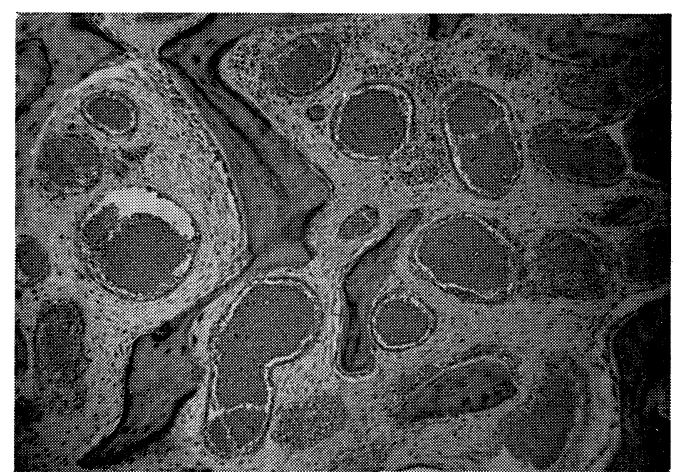

Fig. 18. Severe bleeding in the cavity of dental pulp 


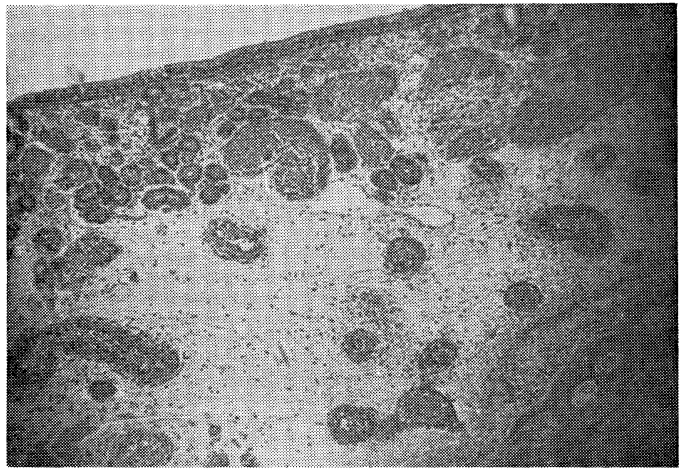

Fig. 19. Hyperemia and edemic change in the accessory nasal membranes

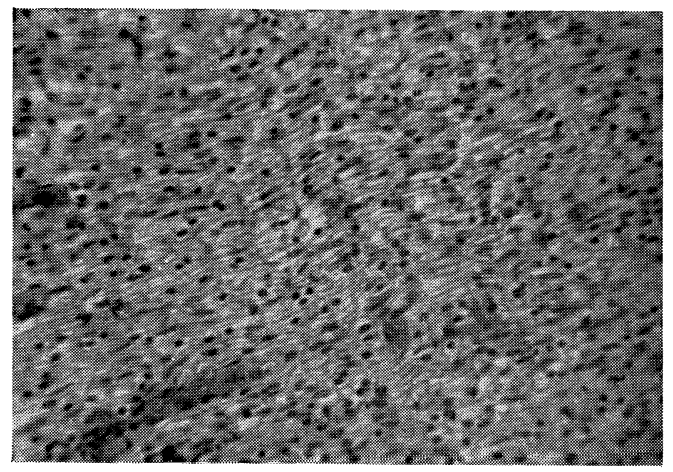

Fig. 20. Nerve fibers

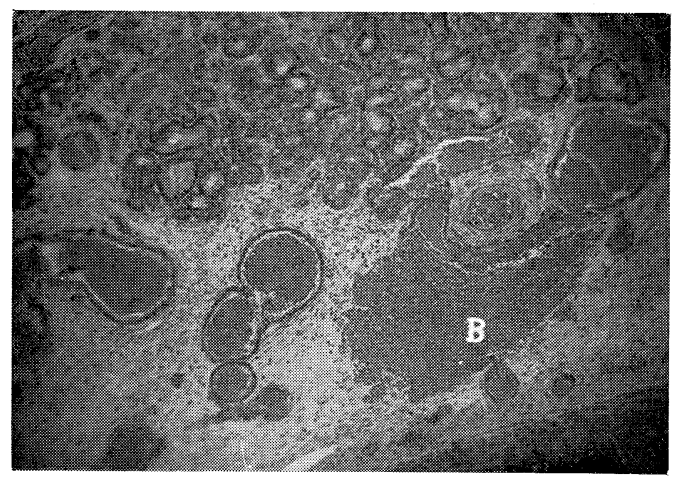

Fig. 21. Hyperemia and bleeding in the gland tissues of angioma but the cover of mucous membranes was not affected (Fig. 19).

9. The nerve fibers and gland tissues.

There were no changes except for a degree of hyperemia (Figs. $20,21)$

\section{Discussions and Consideration}

The accepted idea as regards the body anomaly is usually a congenital one and its developmental mechanism is sought in accidental factors in the process of cell division or in hereditary factors. It is also known that virus infections such as rubeola and influenza when a woman is pregnant, nutritional insufficiency, ill-effect of drugs, irradiation, or follicular hormone are concerned in the picture.

An anomaly owing to thalidomide first came to attract attention as a result of its large incidence in 1958 and it is regarded as an embryopathy of unprecedented character, where it is accompanied with an anomaly or loss of upper limbs, thumbs or lower limbs featured by nevus vascularis. In order for this anomaly to take place, a germinating factor will have to affect the fetus anywhere from 4 to 8 weeks to the impregnation and for this reason, it is due to the use of this thalidomide preparation in the early stage of pregnancy. The subject under discussion in the present paper took the preparation about three weeks of her impregnation, i. e., in the "kritisch phase" according to the term by LENZ and KNAPP [2]. She also took the same preparation in the theoretical period computed by PLIESS [3], which is a period from the twenty-ninth to thirty-ninth day of the impregnation.

An animal experimentation with thalidomide preparation was first undertaken by 
G. F. Somers [4] or England in 1962, who succeeded in producing a deformity case in a female rabbit early in pregnancy similar to that in man. Although an exact mechanism of thalidomide in the production of a deformity is yet to be determined, it is generally inferred, from the findings of animal experiments and clinical examinations, as working strong influence on the formation of organ primoridia, particularly those in the mesoderm system. PLIESs [3] has stated that Wiedemann syndrome which is a typical defect of limbs probably occurs in the scleroblastema period, i. e., 28 th to 42 nd day of the impregnation, which corresponds to a period when noxious factors work on the fetus. As regards to malformation other than that of limbs typical of thalidomide effects, he is inclined to the view that it is not due to the disturbance of organ primordia but to that of critical determination phase in the mesenchymal derivatives. In a special lecture delivered before the 16 th General Session of Japan Medical Congress (Osaka, 1963), Prof. F. Buechner has given his belief that in a thalidomide-baby the malformation is caused by its inhibition of cell-respiration.

On the other hand, it has been known that among the patients who took a large dose of thalidomide there were observed oral diseases such as glossitis which is often caused by Vitamin B-complex. Nervous diseases for which thalidomide is responsible are similar in their nature to those symptoms accounted for by the insufficiency of Vitamin B. These facts seem to point out to an assumption that thalidomide may participate in the disturbance of Vitamin B metabolism [5]. Particularly, rivoflavin in $\mathrm{B}$ family possesses a chemical structure which is similar to that of thalidomide in that they both have -CO-NH-CO-. It is also inferred that thalidomide may work antagonistically on Vitamin $B_{2}$ and thus cause $B_{2}$-avitaminosis. Response of fetuses in the rodent family to $B_{2}$-avitaminosis is actually known to be more sensitive to the skeletons than to the internal organs and other malformations, thus another point of similarity in the thalidomide response in man.

One of differences observable between malformation attributable to thalidomide and what is caused by an insufficiency of $\mathrm{B}_{2}$ or nicotinic acid amid is said to be that in the former there will take place no fissum palatum[5]. In the present case, no fissum palatum was observed. However, this point of difference does not seem to be conclusive enough evidence, as attested by the foregoing findings, to differentiate one from another.

At any rate, various explanations hitherto proposed concerning the malformational mechanism of thalidomide are yet in the realm of surmise. Although many clinical reports on the malformation attributable to thalidomide are published rather abundantly their pathological examinations are rather scarce and this is particularly true of dental area.

In our present pathological examination, what was found to be predominant were disturbances of circulation in the tooth germs and related tissues such as hyperemia, bleeding and edemic changes, other changes having been brought about secondarily by these pathologic changes.

The thalidomide preparation used by the subject under discussion was a mixture of thalidomide with bromvalerilurea, hydroxyalminium and magnesia oxydum for the purposes of sedative, hypnotica and anatida respectively. Thalidomide which is its chief constituent works selectively on the parasympathetic nervous system as a blocking dosage of the said nervous system.

Teratogenesis of thalidomide which allegedly inhibits the cell-respiration of fetus 
as propounded by Prof. Buechner awaits further investigation for more conclusive evidence.

\section{Conclusion}

Our investigation of a thalidomide-baby born of a woman who was in the habit of taking a thalidomide preparation in her pregnancy has revealed the following facts relative to dental pathology.

1) The formation of tetragenic teeth was evinced.

2) Most pathologic changes were disturbances of circulation in the tooth germs and surrounding tissues and as individual cases, desquamation and degeneration of enamel epithelium were apparent.

\section{References}

[1] LENZ, W.: Thalidomide and Congenital Abnormalities, Lancet, 7219, 45 (1962).

[2] LENZ, W. and KNAPP, K. : Die Thalidomid-Embryopathie, Dtsch. med. Wschr., 24, 1232$1242(1962)$

[3] Pliess, G. : Thalidomide and Congenital Malformation, Lancet, 7239, 1128-1129 (1962).

[4] Somers, G. F. : Thalidomide and Congenital Abnormalities, Lancet, 7235, 912-913 (1962).

[ 5 ] IsHII, HideO : Drugs and Abnormalities (in Japanese), Medical Culture, 3, 484-501 (1963). 